\title{
Full Endoscopic Suprapubic Subcutaneous Access: A New Minimally Invasive Surgical Technique for Midline Ventral Hernias
}

\author{
Juan Bellido Luque, MD \\ Biliopancreatic Surgical Division, Gastrointestinal Surgery, Hospital Universitario Virgen Macarena, \\ Seville, Spain. \\ E-mail: j_bellido_|@hotmail.com \\ Beatriz Bascuas Rodrigo, MD \\ Surgical Division, Gastrointestinal Surgery, Hospital Universitario Virgen Macarena, Seville, Spain.

\section{Inmaculada Sanchez-Matamoros, MD} \\ Biliopancreatic Surgical Division, Gastrointestinal Surgery, Hospital Universitario Virgen Macarena, \\ Seville, Spain.
}

Fernando Oliva Mompean, MD

Surgical division, Gastrointestinal Surgery, Hospital Universitario Virgen Macarena, Seville, Spain.

\section{Angel Nogales Muñoz, MD}

Biliopancreatic Surgical Division, Gastrointestinal Surgery, Hospital Universitario Virgen Macarena, Seville, Spain.

(c) Mary Ann Liebert, Inc. DOI: 10.1089/vor.2020.0646
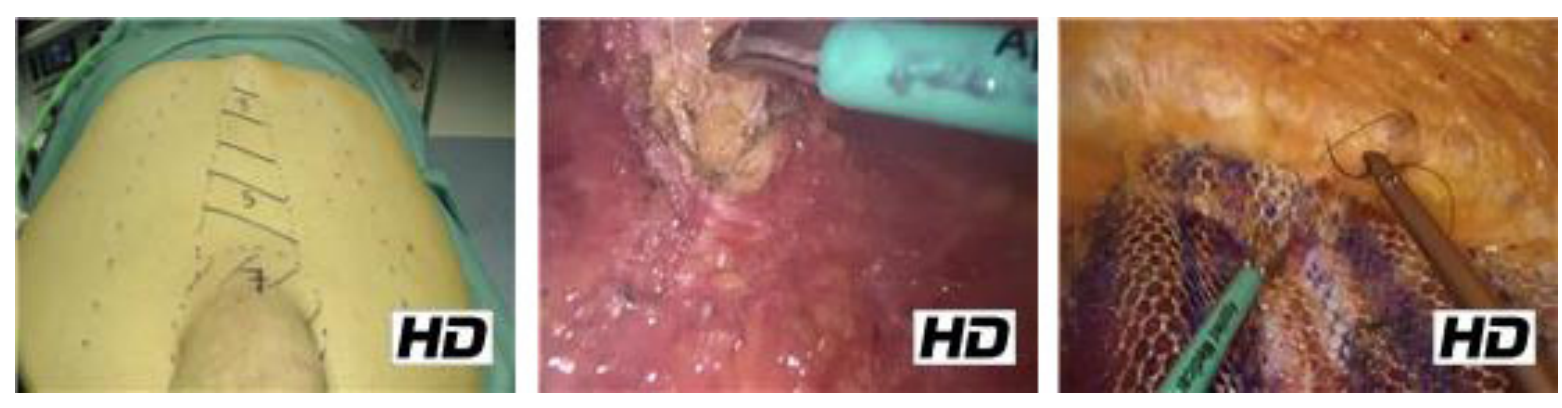

\begin{abstract}
Introduction: When primary ventral hernia and simultaneous diastasis recti are diagnosed, there is no consensus among the surgical community on the surgical treatment regarding indications or surgical technique. However, if diastasis recti are symptomatic or associated with midline hernias, corrective surgery of both pathologies at the same time may be recommended. This video shows a minimally invasive access using an endoscopic Chevrel technique that does not penetrate the peritoneal cavity and avoids intra-abdominal mesh placement. This technique resolves both pathologies simultaneously as well.
\end{abstract}

Materials and Methods: A 50-year-old man presents with a symptomatic $6 \mathrm{~cm}$ umbilical hernia with an associated diastasis rectus. A full endoscopic minimal invasive suprapubic repair was proposed. A suprapubic incision for an $11 \mathrm{~mm}$ trocar and two lateral $5 \mathrm{~mm}$ trocars was placed. A subcutaneous space was developed using $8 \mathrm{~mm} \mathrm{Hg} \mathrm{CO}$ pressure. Subcutaneous dissection was performed exposing the anterior rectus sheath bilaterally while reducing the ventral hernia sac. A complete suprafascial dissection was performed and the anterior layer of the rectus sheath was exposed on both sides from the xiphoid process to the suprapubic region. An incision is made $3 \mathrm{~cm}$ from the medial margin of the rectus sheath bilaterally from the xiphoid process to the subumbilical area, 
exposing both rectus muscles. The medial segments of the anterior rectus sheath were plicated using nonabsorbable barbed suture. This corrected the ventral hernia defect and the rectus diastasis without tension and reapproximated the midline to its original position. A $24 \times 12 \mathrm{~cm}$ size low-weight polypropylene mesh was placed and fixed to the external margin of the anterior layers of the rectus sheaths using running nonabsorbable barbed suture. Finally, a suction drain was placed between the mesh and the subcutaneous tissue and the umbilicus is fixed to the mesh with glue.

Results: The patient was discharged 24 hours after surgery without complications. The drain was removed on the 6th postoperative day. The patient wore an abdominal binder for 6 weeks after the operation. At 18 months postprocedure, the patient remains asymptomatic without any complication or radiologic recurrence.

Conclusions: Totally endoscopic subcutaneous ventral hernia repair with suprapubic access is a new minimally invasive surgical technique that surgically corrects a hernia defect along with an associated diastasis rectus. This technique does not penetrate the intra-abdominal space, thereby avoiding placement of the prosthesis in contact with the viscera and possible sequelae including adhesions or fistulae. No traumatic fixation is needed. The main disadvantage of this technique is the seroma formation and the risks of injury to perforators and lateral nerves.

No competing financial interests exist.

Runtime of video: 9 mins 58 secs

Keywords: ventral hernia repair, minimally invasive ventral/incisional hernia, suprapubic subcutaneous midline hernias

\section{Cite this video}

Juan Bellido Luque, Beatriz Bascuas Rodrigo, Inmaculada Sanchez-Matamoros, Fernando Oliva Mompean, Angel Nogales Muñoz, Full Endoscopic Suprapubic Subcutaneous Access: A New Minimally Invasive Surgical Technique for Midline Ventral Hernias, Videoscopy. 2020, DOI: 10.1089/vor.2020.0646.

Original Publication Date: 2020 